\title{
MRI EVALUATION OF MUSCULOSKELETAL TUMOURS AND ITS CORRELATION WITH HISTOPATHOLOGICAL EXAMINATION
}

\author{
Gautham M.1, Sindhu N.2, Bysani Swaroop ${ }^{3}$, Parthasarathi A. ${ }^{4}$
}

${ }^{1}$ Associate Professor, Department of Radiodiagnosis, Rajarajeswari Medical College and Hospital, Bangalore, Karnataka, India. ${ }^{2}$ Assistant Professor, Department of Radiodiagnosis, Rajarajeswari Medical College and Hospital, Bangalore, Karnataka, India. ${ }^{3}$ Assistant Professor, Department of Radiodiagnosis, Rajarajeswari Medical College and Hospital, Bangalore, Karnataka, India. ${ }^{4}$ Associate Professor, Department of Radiodiagnosis, Rajarajeswari Medical College and Hospital, Bangalore, Karnataka, India. ABSTRACT

\section{BACKGROUND}

Medical imaging revolutionized both diagnostic and therapeutic approaches in musculoskeletal oncology by providing accurate information about the tissue composition and the anatomical relationships of musculoskeletal tumours that is used in tumour detection, staging, therapeutic monitoring, and post therapy surveillance. Radiographs provide critical information regarding lesion location, margin, matrix, mineralisation, cortical involvement and adjacent periosteal reaction. We wanted to determine the role of Magnetic Resonance Imaging in prospective evaluation of patients with clinical suspicion of musculoskeletal tumours. It's relevance as an investigative modality in musculoskeletal tumours and to correlate the findings with final diagnosis by histopathological results.

\section{METHODS}

Prospective diagnostic study conducted with a sample size of 40 suspected musculoskeletal tumour cases in the department of radio-diagnosis, Rajarajeswari Medical College using Siemens Avento 1.5T MRI machine. Both plain radiograph \& Magnetic Resonance Imaging evaluation were done \& further diagnosis was confirmed by histopathological examination. All patients presenting with localized swelling were included in the study. Histopathology/FNAC is a mandatory criterion as proof for final diagnosis. Patients with generalized oedema, presenting with recurrence of a primary lesion and patients in whom MRI was contraindicated e.g.: with pacemakers, metallic implants, were excluded.

\section{RESULTS}

For the initial evaluation of musculoskeletal tumours, radiographs should be the first line of imaging, as they are relatively inexpensive. It helps in diagnosis, separating benign from malignant cases and helps in deciding the requirement of additional imaging examination by Magnetic Resonance Imaging. Soft tissue-, joint- \& neurovascular-involvement is better evaluated by MRI $\&$ helps to a greater extent in planning the course of treatment.

\section{CONCLUSIONS}

A combination of Radiography \& Magnetic Resonance Imaging evaluation gives accurate \& all-round information regarding the musculoskeletal tumours, increasing the sensitivity \& specificity to a much higher extent than if done individually.

\section{KEY WORDS}

Radiograph, Magnetic Resonance Imaging, Benign, Malignant, Bone Tumour

HOW TO CITE THIS ARTICLE: Gautham M, Sindhu N, Swaroop B, et al. MRI evaluation of musculoskeletal tumours and its correlation with histopathological examination. J. Evolution Med. Dent. Sci. 2019;8(30):2398-2402, D0I: $10.14260 /$ jemds/2019/525

\section{BACKGROUND}

Medical imaging revolutionized both diagnostic and therapeutic approaches in musculoskeletal oncology by providing accurate information about the tissue composition and the anatomic relationships of musculoskeletal tumours that is used in tumour detection, staging, therapeutic monitoring, and post therapy surveillance.

Radiographs provide critical information regarding lesion location, margin, matrix, mineralisation, cortical involvement

'Financial or Other Competing Interest': None.

Submission 29-04-2019, Peer Review 08-07-2019,

Acceptance 15-07-2019, Published 29-07-2019.

Corresponding Author:

Dr. Parthasarathi A.,

Associate Professor, Department of Radiodiagnosis,

Rajarajeswari Medical College and Hospital,

Kambipura, Mysore Road, Bangalore-560074,

Karnataka, India.

E-mail:dr.pathu@gmail.com

DOI: $10.14260 /$ jemds $/ 2019 / 525$ information than any other imaging modality in the study of bone lesions \& remains the cornerstone for the differential diagnosis of skeletal tumours and tumour like lesion, or at least narrow the diagnostic possibilities, include patterns of bone destruction, lesion margins, internal characteristics of the lesion, type of host bone response, location, site and position of the lesion the skeletal nature. The radiographic features coupled with clinical information helps define whether the lesion is neoplastic or nonneoplastic, primary or metastatic and will help further direct the subsequent work up. Magnetic Resonance Imaging (MRI) is highly sensitive to the presence of musculoskeletal soft-tissue lesions and also provides exquisite definition of their features. Although there are few, if any, pathognomonic findings for malignant or benign lesions in diagnostic imaging, a high degree of confidence or statistical likelihood can be achieved in many instances using MR imaging. ${ }^{2}$ 
MRI is the best modality for focal extent and local staging. ${ }^{3}$ This is particularly true with regards to invasion of muscle, neurovascular structures and adjacent fat planes and the degree of marrow involvement. MRI has also been shown to be superior in assessing intra-articular extension and the presence of intra-tumoural necrosis and haemorrhage. MRI is particularly appropriate if it is necessary to show soft tissue changes within an area of sclerosis, which may be responsible for the new bone formation and which may be masked on radiographs and CT by the surrounding sclerosis. ${ }^{4} \mathrm{MRI}$ is the best technique to detect skip lesions which are often missed on other imaging means. ${ }^{5}$ In the follow up, MRI is the best single method to assess the response to therapy and to detect tumour persistence. ${ }^{6}$ Particular emphasis is on those soft tissue and bone diagnoses that may be confidently made or suggested by MRI and lesions that are frequently encountered as incidental findings on examinations obtained for unrelated reasons. The use of MRI in differentiating benign from malignant soft tissue lesions, follow-up evaluation for differentiation of recurrent tumours from postoperative or radiation change, and response to therapy are also covered. Imaging evaluation for diagnosis and staging should be done before biopsy. Biopsy is the definitive diagnostic procedure and should be carried out only after the appropriate diagnostic and staging tests. Whenever a bone lesion is suspected, clinico-radiological and pathologic correlation is essential to make a more accurate diagnosis and improve patient care. ${ }^{7}$

\section{METHODS}

Prospective diagnostic study having sample size of 40 patients for a period of 2 years at Rajarajeswari medical college \& hospital, Department of Radio-Diagnosis using Siemens Avento 1.5T MRI machine.

\section{Inclusion Criteria}

- All patients presenting with localized swelling/pain.

- Histopathology/Fine needle aspiration cytology (FNAC) a mandatory criterion as proof for final diagnosis.

\section{Exclusion Criteria}

- Patients with generalized oedema.

- Patients presenting with recurrence of a primary lesion.

- Patients in whom MRI was contraindicated e.g.: With pacemakers, metallic implants.

- Undiagnosed suspected cases of primary musculoskeletal tumours which were diagnosed as metastasis or inflammatory/infective aetiology on histopathological examination were excluded from the study.

\section{RESULTS}

\section{Age Distribution}

Patients of all age groups were included in the study. The youngest patient was 6-year-old and the oldest was 75 years old. Maximum number of patients were in the age group 1130 years.

\section{Sex Distribution}

Out of 40 patients, 22 (55\%) were males and 18 (45\%) were females. Sixth decade was the commonest age group in males and second and fifth decade was commonest age group in females for musculoskeletal tumours.

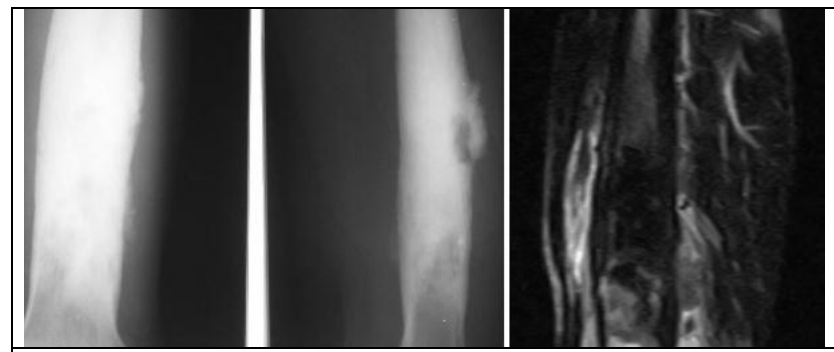

Figure 1. Radiograph and MRI Image of Osteosarcoma

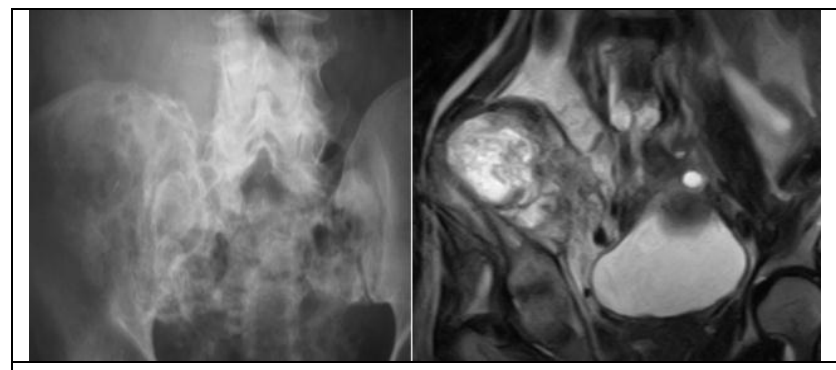

Figure 2. Radiograph and MRI Image of Chondrosarcoma

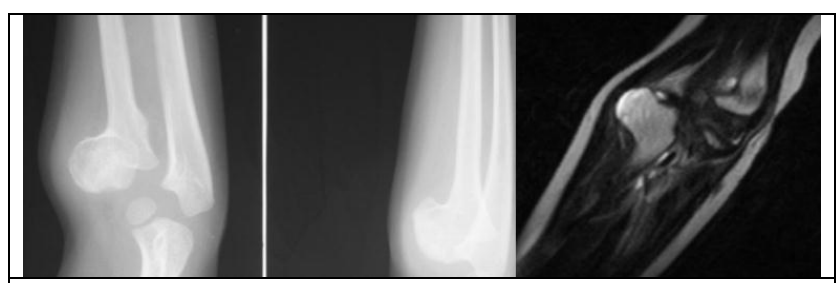

Figure 3. Radiograph and MRI Image of Osteochondroma

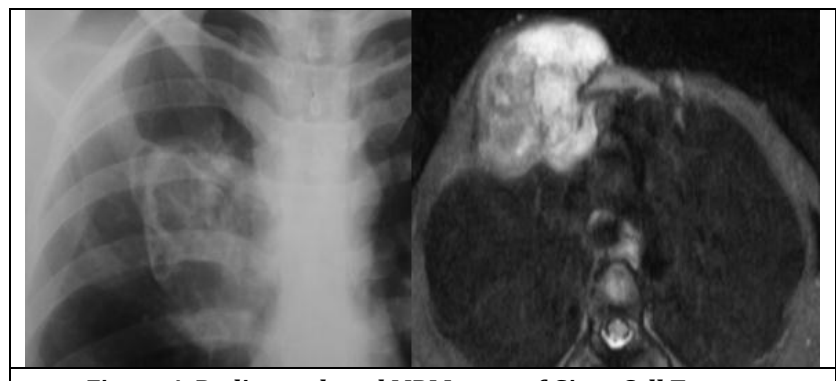

Figure 4. Radiograph and MRI Image of Giant Cell Tumour

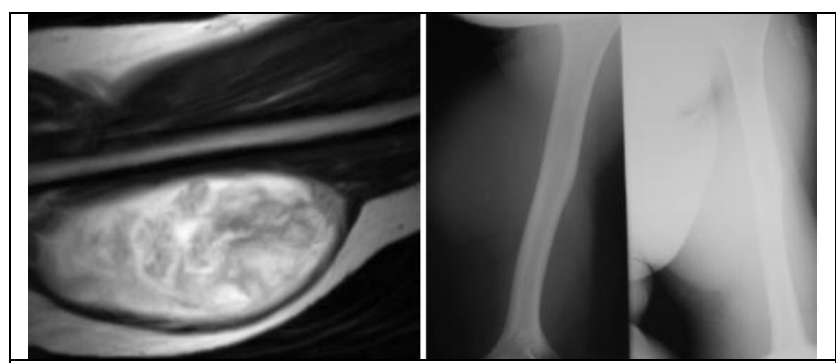

Figure 5. Image Showing MRI and Radiograph of Malignant Fibrous Histiocytoma

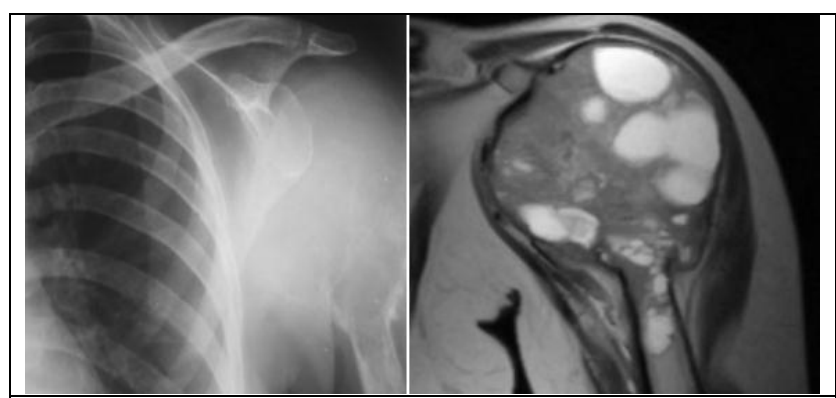

Figure 6. Image Showing Radiograph and MRI of Aneurysmal Bone Cyst 


\section{Location of Lesion}

Appendicular skeleton was involved in 15 patients (37.5\%). Axial skeleton was involved in 2 patients (5\%) and soft tissue in 23 patients $(57.5 \%)$.

\section{Demographic Profile}

The demographic profile of the patients revealed pain and swelling to be the most common presenting symptoms.

\section{Nature of Lesion}

Twenty-two patients (55\%) had benign musculoskeletal tumours and 18 patients (45\%) had malignant musculoskeletal tumours.

\section{Zone of Transition}

Narrow, if it is so well defined that it can be drawn with a fine-point pen. Wide, if it is imperceptible and cannot be drawn at all. Eight patients had narrow zone of transition and 4 patients had wide zone of transition on Radiographs whereas on MRI, 3 patients had narrow zone of transition and 8 patients had wide zone of transition.

\section{Soft Tissue Involvement}

Soft tissue involvement was depicted in 18 patients on radiographs $(45 \%)$ whereas MRI demonstrated soft tissue involvement in 24 patients (60\%) and surgery demonstrated soft tissue involvement in 25 patients (62.5\%).

\section{Adjacent Joint Involvement}

Radiographs showed adjacent joint involvement in 1 patient (2.5\%). MRI demonstrated adjacent joint involvement in 4 patients $(10 \%)$ and surgery demonstrated adjacent joint involvement in 5 patients (12.5\%). In our study, osteosarcoma was the most frequent malignant primary bone tumour ( 5 cases-16\% of total cases). However instead of benign chondroid lesions, Giant cell tumour was the most common benign primary bone tumour in our series of 40 patients ( 4 patients $-8 \%$ of all cases). This may be because most of the patients with GCT came for further imaging and surgical intervention.

The following imaging features of musculoskeletal tumours were evaluated using MRI-

- Zone of transition.

- Soft tissue involvement.

- Adjacent joint involvement.

- Neurovascular bundle involvement.

- Signal Intensity pattern.

\section{DISCUSSION}

\section{Osteosarcoma}

Out of Five cases of Osteosarcoma, femur was the most commonly involved bone. The age group varied from 51-55 years. They were seen predominantly in females (M: $\mathrm{F}=2: 3$ ).

- Radiographs showed mixed density metaphyseal lesion with wide zone of transition with soft tissue components, ossification and periosteal reaction (Sunray appearance and Codman's triangle).

- The lesions were heterogeneously hypointense on T1W and $\mathrm{T} 2 \mathrm{~W}$ images.
MRI was better in delineating the adjacent joint involvement. There was soft tissue involvement in all cases $(100 \%)$. Codman's type of periosteal reactions was seen in two patients, sunray type of periosteal reaction was seen in one patient. Cortical break was seen in all patients (100\%). Adjacent joint involvement was present in two cases.

\section{Osteoid Osteoma}

There was 1 case of Osteoid osteoma in present study. The average age of presentation was 12 years and the incidence was (M: $F=1: 0)$. The lesion showed the following features on imaging:

- Radiographs- AP and lateral view demonstrated sclerosis and thickening of the cortex with a lucent nidus within it

- MRI also demonstrated the sclerosis of cortex with nidus formation.

- On MRI the nidus was seen as a hyperintense focus within the thickened cortex on T2W sequences with minimal bone marrow oedema in one patient. These findings confirmed diagnosis of osteoid osteoma.

\section{Chondrosarcoma}

There was one patient of chondrosarcoma in the present study. The age of presentation was 45 years. Females were more commonly involved than males ( $\mathrm{M}: \mathrm{F}=0: 1$ ).

- Radiograph demonstrated mixed density lesion with wide zone of transition and specks of calcification.

- On MRI, the lesions were isointense on T1W, heterogeneously hyperintense on T2W. MRI was better in delineating the adjacent joint involvement and NVB involvement. On imaging diagnosis of chondrosarcoma was given which was confirmed on biopsy.

\section{Osteochondroma}

There were 3 cases of Osteochondroma in present study. The average age of presentation was 12 years. Males were more commonly involved than females ( $\mathrm{M}: \mathrm{F}=2: 1)$. The lesion showed the following features on imaging.

- Radiographs demonstrated that bony pedunculated outgrowths whose cortex and medulla were continuous with that of bone of origin.

- MRI demonstrated continuity of cortex and medulla with that of lesion.

MRI demonstrated a high signal intensity cap on T2W images and thickness of cap was less than $1 \mathrm{~cm}$ in all three cases.

\section{Giant Cell Tumour}

There were 4 cases of GCT in our study. They occurred in age group of 23-47 years. They presented with swelling. Male to female ratio was 1:3.

- Radiographs showed an eccentric expansile lytic extra articular lesion in meta-epiphysis of the bones.

- On MRI, the lesions were homogenously hypointense on T1W. Varied appearances like heterogeneously hyperintense with fluid filled areas and necrotic areas were seen on $\mathrm{T} 2 \mathrm{~W}$.

- MRI showed joint involvement in two cases (50\%). Soft tissue involvement is seen in one case $(25 \%)$. 


\section{Chordoma}

One case was present in our study. Ages of patients were between 35-40 years.

- Radiographs showed a lytic lesion of sacrum and coccyx with wide zone of transition. Calcification was present within large soft tissue mass.

- On MR imaging the lesion was heterogeneously hypointense on T1W images and heterogeneously hyperintense on $\mathrm{T} 2 \mathrm{~W}$ images with hypointense foci on all sequences suggestive of calcification.

Adjacent soft tissue involvement and bilateral sacroiliac joints involvement was seen in one case.

\section{Haemangioma}

There was one case of haemangioma in present study. A 52year old male patient presented with backache for 6 months.

- Radiograph demonstrated a radiolucent lesion with fine vertical striations involving L2 vertebral body.

- On MRI, the lesion is isointense to muscle with fine delicate strands on T1W and hyperintense on T2Wimaging. The case was not operated.

Vascular lesions of bone are commonly seen incidentally at radiology. The most common sites of involvement are the vertebrae and skull. At MR imaging, vertebral haemangiomas demonstrate the classic vertical trabecular or radiating pattern of thickening seen at radiography, with high signal intensity on T1-and T2weighted images due to the presence of intratumoural fat. Similar appearances were seen in the present study.

\section{AV Malformation}

One case of AV malformation was examined aged 26 yrs., female patient. These rare vascular malformations result from abnormal vessel development, which bypasses the capillary bed. MR imaging identifies high flow as signal voids on spin-echo imaging or as increased signal with gradientrecalled echo. ${ }^{8}$ An arteriovenous malformation confined to a muscle sheath may resemble a vascular tumour. ${ }^{9}$ The skin may appear thickened and bony involvement results in lytic changes with cortical thinning. Treatment is trans arterial hyper selective embolization ${ }^{10}$

\section{Nonossifying Fibroma}

There were two cases of nonossifying fibroma in our study. A 45-year old female patient presented with swelling in left leg.

- On radiograph, there was a lucent area involving metaphysis of left tibia with well-defined margins and surrounding zone of reactive sclerosis.

- $\quad$ On MRI, the lesion was homogenously hypointense on TIW and T2W images.

The case was confirmed on biopsy as non-ossifying fibroma. A nonossifying fibroma (Sometimes referred to as a fibrous metaphyseal-diaphyseal defect or fibrous medullary defect) is considered to be an enlarged benign cortical defect. However, the lesion is mostly intramedullary, and the cortical involvement is not always present. Most lesions occur in the bones of the lower extremity, especially about the knee. On radiographs, the lesion is seen as an elongated, well- marginated, osteolytic, intramedullary defect in the metaphysis of a long bone often with a multilocular appearance ${ }^{11}$. Similar pattern was observed in the present study.

\section{Malignant Fibrous Histiocytoma}

4 cases with age frequency of 51-55 yrs. were evaluated.

- On radiograph, there was a metaphyseal lesion involving proximal radius with wide zone of transition with soft tissue component and calcification.

- On MRI, the lesion was heterogeneously hypointense on T1W and T2W images.

- The lesion involved the epi-meta-diaphysis of proximal radius with soft tissue component, growth plate involvement and involvement of elbow joint by tumour tissue. Muscles of forearm and neurovascular bundle were also involved.

\section{Malignant Mesenchymal Tumour}

There were eight cases of malignant mesenchymal tumours. Age group varied from 29-65 years. They were seen predominantly in females (M: $\mathrm{F}=3: 5$ ).

- On MRI, the lesions were hypointense to isointense on T1W images and predominantly hyperintense on T2W. Areas of haemorrhage were seen in 4 cases (50\%). Areas of necrosis was seen in all cases (100\%). Specks of calcification were seen in 2 cases (25\%). Adjacent joint was involved in 3 cases (37.5\%). Neurovascular bundle was involved in 4 cases (50\%)

On imaging, a diagnosis of malignant mesenchymal tumour was made. The lesion was excised, and biopsy confirmed malignant mesenchymal tumour as the final diagnosis.

\section{Synovial Sarcoma}

In this study there were 2 cases of synovial sarcoma between the age groups of $17-40$ yrs., both were female patients. This malignant, high-grade tumour is not derived from the synovial membrane but from primitive mesenchymal cells, differentiated into spindle cells and sometimes epithelial cells. Although classically located near a joint, it can occur anywhere. Nearly half of cases occur in children and adolescents 12, and it is the most common nonrhabdomyosarcoma soft tissue sarcoma to occur in this age group; however, it constitutes only $5 \%$ of all paediatric soft tissue sarcomas ${ }^{13}$. Prognosis is best if the tumour is less than $5 \mathrm{~cm}$ or if it develops in a younger child around the hand, foot, or knee. More common in the lower extremities than in the upper and especially common around the knee. Synovial sarcoma usually occurs in the deep compartment, often within $7 \mathrm{~cm}$ of a joint. ${ }^{14}$ Although it appears to displace rather than invade adjacent structures. At surgery there usually is microscopic invasion beyond the pseudocapsule. On T1W imaging it appears isointense to muscle and on T2W imaging it appears heterogeneous but hyperintense. ${ }^{15}$ Haemorrhage may be present and fluid-fluid levels are seen in approximately $20 \%$ of cases. This tumour may appear purely cystic and has been mistaken for a Baker's cyst, hematoma or ganglion cyst. It is the most common malignant tumour to be mistaken as benign. ${ }^{16}$ Complete resection is important and re- 
resection is often required. Local radiation therapy may help if surgical margins are small.

\section{Aneurysmal Bone Cyst}

There were two cases of aneurysmal bone cyst in present study. The age of patient was 16-27 years and was male.

- The lesion was in greater tuberosity of humerus. The lesion was located in metaphysis. Radiograph showed expansile lytic lesion and narrow zone of transition without periosteal reaction.

- On MRI, fluid-fluid level was seen, which was characteristic of aneurysmal bone cyst.

\section{Simple Bone Cyst}

One patient with simple bone cyst in present study. A 19-year old boy presented with right foot pain and another. Radiographs showed a solitary lucent lesion. MR imaging showed a hypointense lesions on T1W image and hyperintense lesions on T2W image. Simple or unicameral bone cysts are fluid containing lesions that are typically seen in children and young adults. Flat bone lesions are considerably less common and are typically seen in older patients. In the flat bones, cysts are common in the anterior part of calcaneum and ilium. MRI will show a lesion with signal intensity similar to that of fluid, with high signal intensity on fluid sensitive image (Similar to present study). The fibrovascular tissue in the lining of the lesion may show distinct enhancement after contrast administration. ${ }^{17} \mathrm{~A}$ fallen fragment may be seen in the dependent part of the cyst after fracture through the cyst. ${ }^{18}$ At MR imaging the fluid-filled cyst is high signal on T2 weighted images and intermediate signal on T1 weighted images. Simple bone cysts do not demonstrate fluid-fluid levels ${ }^{19}$. ABC are usually septated and after gadolinium administration enhancement of the septations and "cyst" walls may be seen.

\section{CONCLUSIONS}

Present study showed that for the initial evaluation of musculoskeletal tumours, radiographs should be the first line of imaging. Not only are radiographs relatively inexpensive but the differential diagnosis of most primary musculoskeletal tumours is generated based on features detected on radiographs. X-ray can differentiate benign from malignant lesions and allows one to decide whether additional imaging examinations should be performed. Magnetic Resonance Imaging is the mode of choice for evaluation of musculoskeletal tumours. It is highly specific \& sensitive in diagnosing musculoskeletal tumours. Its combination with conventional radiograph leads to better analysis \& accuracy. It gives added information of surrounding tissues including joints \& neurovascular bundle. A combination of Radiograph \& MRI evaluation gives accurate \& all-round information regarding the musculoskeletal tumours, increasing the sensitivity \& specificity to a much higher extent than if done individually.

\section{REFERENCES}

[1] Baweja S, Arora R, Singh S, et al. Evaluation of bone tumours with Magnetic Resonance Imaging and co relation with surgical and pathological findings. Ind J Radiol \& Imag 2006;16(4):611-8.
[2] Weatherall PT. Benign and malignant masses. MR imaging differentiation. Magn Reson Imaging Clin N Am 1995;3(4):669-94.

[3] Reiser MF. MRI in diagnosis and staging of bone tumours. AJR 1996;132:567-76.

[4] Holland BR, Freyschmidt J. Magnetic resonance tomographic results in bone changes associated with sclerosis. Rofo 1988;149(5):513-21.

[5] Schima W, Amann G, Stiglbauer R, et al. Preoperative staging of osteosarcoma: efficacy of MR imaging in detecting joint involvement. AJR 1994;163(5):1171-5.

[6] Balzarini L, Sicilia A, Ceglia E, et al. Magnetic resonance in primary in primary bone tumours: a review of 10 years of activities. Radiol Med 1996;91(4):344-7.

[7] Priolo F, Cerase A. The current role of radiography in the assessment of skeletal tumours and tumours and tumour-like lesions. Eur J Radiol 1998;27(1):S77-85.

[8] Frouge C, Vanel D, Coffre C, et al. The role of magnetic resonance imaging in the evaluation of Ewing sarcoma. A report of 27 cases. Skeletal Radiol 1988;17(6):387-92.

[9] Snug MS, Lee GK, Kang HS, et al. Sacrococcygeal chordoma: MR imaging in 30 patients. Skeletal Radiology 2005;34(2):87-94.

[10] Enjolras 0. Classification and management of the various superficial vascular anomalies: hemangiomas and vascular malformations. J Dermatol 1997;24(11):701-10.

[11] Vlychou M, Athanasou NA. Radiological and pathological diagnosis of paediatric bone tumours and tumour-like lesions. Pathology 2008;40(2):196-216.

[12] Woertler K. Benign bone tumours and tumour-like lesions: value of cross-sectional imaging. Eur Radiol 2003;13(8):1820-35.

[13] Mahnken AH, Nolte-Ernsting CC, Wildberger JE, et al. Aneurysmal bone cyst: value of MR imaging and conventional radiography. Eur Radiol 2003;13(5):1118-24.

[14] Konez 0, Burrows PE. Magnetic resonance of vascular anomalies. Magn Reson Imaging Clin N Am 2002;10(2):363-88, vii.

[15] Meyer JS, Hoffer FA, Barnes PD, et al. Biological classification of soft-tissue vascular anomalies: MR correlation. AJR Am J Roentgenol 1991;157(3):559-64.

[16] Wetzel LH, Levine E, Murphey MD. A comparison of MR imaging and CT in the evaluation of musculoskeletal masses. Radiographics 1987;7(5):851-74.

[17] Berquist TH, Ehman RL, King BF, et al. Value of MR imaging in differentiating benign from malignant softtissue masses: study of 95 lesions. AJR Am J Roentgenol 1990;155(6):1251-5.

[18] Miser JS, Pizzo PA. Soft tissue sarcomas in childhood. Pediatr Clin North Am 1985;32(3):779-800.

[19] McCarville MB, Spunt SL, Skapek SX, et al. Synovial sarcoma in pediatric patients. AJR Am J Roentgenol 2002;179(3):797-801. 